\title{
The investigation of the changes in the surface glycoconjugates using two different spheroid models of breast cancer cells and availability assessment of these spheroid models for rapid diagnosis
}

\author{
Yosun MATER ${ }^{1, *(i)}$, Günnur DEMIRCAN² (i) \\ ${ }^{1}$ Department of Molecular Biology and Genetics, Faculty of Basic Sciences, Gebze Technical University, Kocaeli, Turkey \\ ${ }^{2}$ Department of Medical Biology and Genetics, Faculty of Medicine, Demiroglu Bilim University, İstanbul, Turkey
}

\begin{abstract}
Received: $14.11 .2020 \quad \bullet \quad$ Accepted/Published Online: 01.02.2021 • Final Version: 23.04 .2021
Abstract

The importance of early cancer diagnosis has led to development of many different diagnostic methods. In this context, the studies investigating the presence and amount of sugar residues to use as indicators in the identification of cancer cell type have become prominent. In the present study, sialic acids found on the membrane surfaces of ER (+) MCF-7 and ER (-) MDA-MB-231 breast cancer cell lines were labeled using threedimensional (3D) cell culture (Spheroid) model as the closest method to the patient sample, thus its natural environment, among the cell culture methods. These sugar units that play a role in regulation of important immune characteristics such as recognition, binding and metastasis were made visualizable by applying fluorescent-labeled lectins such as FITC-(Wheat Germ Agglutinin) specifically binding to sialic acid units (GlcNAc, Neu5Ac) including particularly ß-GlcNAc and FITC-(Maackia Amurensis-Lectin-1) specifically binding to Galß4GlcNAc type sialic acids. These glycan units were specifically labeled with FITC-(Maackia Amurensis-Lectin-1) and FITC- (Wheat Germ Agglutinin) and radiation intensities were analyzed relatively. The two different breast cancer cell cultures were compared with respect to change in the amounts of sialic acid residues containing $\alpha-2,3-$ and $\alpha-2,6$ bonds using fluorescent-labeled lectins. In the present study, we have performed a precise, accurate and rapid determination of the sugar content in the different breast cancer cell surface lines by means of fluorescent-labeled lectins and carried out a relative comparison between the micrographs.
\end{abstract}

Keywords: breast cancer, lectins, sialic acid, spheroid

\section{Introduction}

Breast cancer is the most frequently diagnosed cancer type in women with estimated 1.38 million new cases per year worldwide. Totally 458,000 deaths occur due to breast cancer in both developed and developing countries each year. This number of deaths indicates that cancer is the most common cause of death in women (Eccles et al., 2013). Normal cells have a regular life cycle and limited number of cell divisions. Like all other cancer cells, breast cancer cells are the cells that altered cell division characteristics due to various mutations. This alteration is caused by the differentiated pathways of cell division. These differentiations result from the changes in the cell surface glycans (Kohnz et al., 2016). Sialic acids on the end units of the outer cell surface glycans play an essential role in cellular recognition and interaction processes with other cells and microenvironment. High concentrations of sialic acids may also lead to structural changes both in normal and cancer cell surfaces (Samraj et al., 2014).

The change in the amounts of sialic acid residues particularly containing $\alpha-2,3-$ and $\alpha-2,6$ bonds located on the cell surfaces leads to development of avascular multicellular tumoral formations in normal and cancer cells. It has been demonstrated that they have a capability to form in vivo xenograft tumors particularly in the cancer cell lines. It is considered that the obtainable data will provide a supportive information infrastructure in selection of the appropriate cells, administration of the oriented treatment and establishment of the early diagnosis (Akasov et al., 2016).

Pathological tests are gold standard for diagnosis of cancer. These tests have an important role in identification of etiology and pathogenesis of cancer as well as assessment of clinical correlation and symptoms of the disease. The most novel molecular technologies, particularly high-throughput technologies, have detected that even morphologically similar subtypes of breast cancer may show molecular heterogeneity. For instance, infiltrating ductal carcinoma was found to have at least 4 molecular subtypes. These subtypes were classified 
as luminal (ER+, PR+, Her-2/neu-), Her-2 overexpressing (ER-, PR-, Her-2+), basal-like (ER+, PR-, Her-2-, CK5/6+, EGFR+) and normal breast-like (ER-, PR-, Her-2-) breast carcinomas. Each type shows different clinical results. The proliferative gene expression of these subtypes is detected by immunohistochemical markers including very expensive molecular tests such as Ki67, Her-2, PR and ER (Anthony et al., 2011). Beside all these tests and diagnostic techniques, the importance of glycobiology in cancer research studies has progressively increased in the recent years. Glycosylation may function as a regulator mechanism that can control various pathological processes. The glycosylation disorders in humans may lead to diseases. Human glycomics involves a substantial amount of biological information (Pinho and Reis, 2015). Among the cancer researches carried on cancer cells, the studies investigating the presence and amount of sugar residues to use as indicators in the identification of cancer cell type have become prominent. These studies are conducted using scan techniques such as PET-SCAN and their results are assessed for confirmation by comparing with lectin-labeling (WGA, SNA, MAL etc.) methods (Martinez-Duncker et al., 2011).

Lectins are the glycoproteins with a particular glycanspecific affinity. Glycoprotein enrichment through lectinaffinity is one of the main techniques that utilize specificity of lectins to a particular glycan residue or ligand type. Currently, common use of two different glycoprotein/glycopeptides are accepted as enrichment techniques. For instance, Wheat Germ Agglutinin (WGA) mainly interact with N-Acetyl Glucosamine (GlcNAc) residues that have essentially have a glycan structure. The enriched glycan structures located on the cancer cell surfaces can be visualized by means of the lectins that specifically bind to these glycans (Varki et al., 2017; Schaurer et al., 2018). On the other hand, a multilectin mixture has been used to bind and enrich many various glycans. Lectin enrichment technique were effectively used to analyze the glycoproteomic changes associated with different cancer types including lung, breast, and liver cancers (Song and Mechref, 2015).

In the two-dimensional (2D) cancer cell culture systems, cells are cultured as a single layer on a flat and rigid surface with lacking cell-cell and cell-matrix interactions of natural tumors. These cells with artificial polarity are exposed to a cell skeleton regulation different from original tumor cell microenvironment. That causes abnormal gene and protein expression. Contrarily, three-dimensional (3D) cell culture systems allow culturing cancer cells solely or together with various cell types. This procedure can closely mimic the natural environment of tumors and promote cell-cell and cellmatric interactions. The cells cultured by three-dimensional (3D) models in this way gain morphological and cellular characteristics similarly with in vivo tumors and thereby the closest cancer cell modelling directly to the patient samples can be directly realized. By means of this method, the impact of the microenvironment on the tumor can be evaluated most closely
(Nath and Devi, 2016). For this purpose, the amounts of sugar residues were investigated by implementing immunofluorescent-labeled lectins FITC-[WGA] and FITC[MAL-I] to the spheroids obtained from the MCF-7 [ER (+)] and MDA-MB-231 [ER (-)] spheroid (3D) cancer tissue/cell lines. By this was, it was aimed to demonstrate the availability of this labeling technique in the closest $3 \mathrm{D}$ cell model to the patient cancer tissue. Thus, it would be possible to determine the difference between the sugar profiles of the breast cancer cells.

Sugar residues were primarily preferred in this determination since these units control the recognition and binding mechanisms of the cell. That would facilitate for researchers identifying and clarifying the cells that were obtained from different sources and that have different characteristics. For this purpose, the fluorescent-labeled lectins applied to specifically identify the cell surface sugar lines will be visualized applying the modified staining techniques (Mater and Özdaş, 2018; Demircan and Mater; 2019) as in our previous studies. Thanks to the method used in this breast cancer cell model, a fast, specific and new labeling technique with the help of lectins has been developed. Thus, it was concluded that a more specific and rapid method can be created in the diagnosis of breast cancer.

\section{Materials and methods}

\subsection{The preparation of 3D spheroid culture model for breast cancer cells}

MCF-7 (ATCC ${ }^{\circ}$ HTB-22 ${ }^{\mathrm{TM}}$ ) and MDA-MB-231

(ATCC®HTB-26 ${ }^{\mathrm{TM}}$ ) cell strains supplied from American Type Culture Collection (ATCC) were used in our study. The cells were incubated at $37^{\circ} \mathrm{C}, 5 \% \mathrm{CO}_{2}$ concentration and 1 atm pressure in their medium containing $10 \%$ inactivated fetal bovine serum (FCS, Gibco, Invitrogen Life Science, Paisley, UK)), $100 \mathrm{U} / \mathrm{ml}$ penicillin and $100 \mu \mathrm{g} / \mathrm{ml}$ streptomycin (Sigma-Aldrich, St Louis, MO, USA). Leibovitz's L-15 Medium and Eagle's Minimum Essential Medium were used for MDA-MB-213 and MCF-7 cell strains, respectively.

The cells in semi-confluent flasks were harvested using $0.05 \%$ trypsin (Sigma-Aldrich), centrifuge (Nuve NF200, Laboratory and Sterilization Technology, Ankara, Turkey) following the addition of FCS for trypsin inactivation and then resuspended in culture medium. The cells counted for 3dimensional (3D) spheroid model cell culture were seeded so that each well will contain a cell suspension of $1 \times 10^{3}$ cells $/ \mathrm{ml}$ in the six-well microplates. The wells of the plates were coated with 3\% Noble Agar (Difco Laboratories Inc., BD Diagnostic Systems Detroit, MI, USA) to form a non-adhesive surface. Two weeks after onset, the plates were examined regarding development of spheroid (colonies or spheres). The spheroids from each well were counted under microscope and measured, representative fields were photographed. Labeling was performed using fluorescent-labeled lectins for characterization of the spheroids. 


\subsection{Labeling procedure of the spheroids using lectins}

Fluorescent-labeled lectins were purchased from Vector Laboratories (Burlingame, CA, USA) and prepared to be used according to the manufacturer's usage protocol. In the labeling process, the mediums of the spheroids were removed to maintain spheroids in the six-well plates. The spheroids were washed with PBS after removing mediums. Following this step, spheroids were incubated with fluorescent-labeled lectins for an appropriate period. After incubation, fluorescentcontaining lectin was removed from the spheroids by washing with PBS and spheroids were left to dry for 10 minutes. Following this step, a special mounting solution (Fluoroshield Mounting Medium with DAPI, ab 104139, Abcam) containing DAPI (4' 6-diamidino-2-phenylindole) was dropped onto the spheroids and the samples were examined using NikonEclipse-80i-Fluorescence-Microscope. The obtained micrographs were visualized to be evaluated using NIS Elements BR 3.0 Software provided by the same device.

\section{Results}

MCF-7 and MDA-MB 231 breast cancer cell lines were grown in the three-dimensional (3D) cell culture and spheroids were created in our study (Figs. 1A, 2A). In the next step, MCF-7 [ER (+)] and MDA-MB-231 [ER (-)] spheroids were stained using fluorescent-labeled lectins (Figs. 1B, 1C and Figs. 2B, 2C). Accordingly, sialic acid units (GlcNAc, Neu5Ac) and also glycan units containing particularly ß-GlcNAc type sialic acids were visualized by using FITC-[WGA] while Galß4GlcNAc type glycan units were displayed by applying FITC-[MAL-I] as the specifically binding fluorescent-labeled lectins.

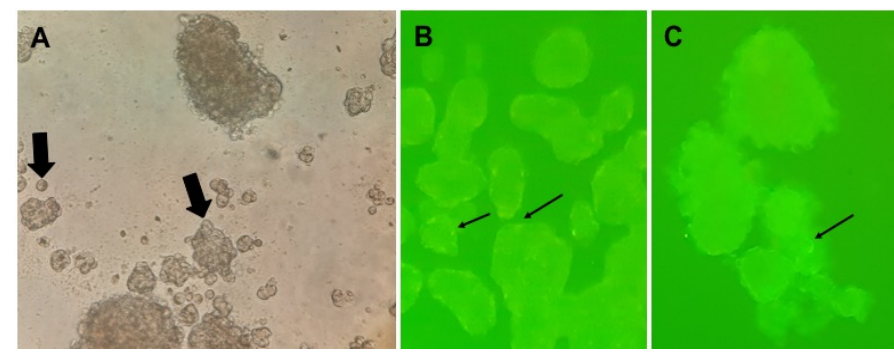

Fig. 1. Cells produced in MCF-7 three-dimensional (3D) cell line, spheroids. Control cells and spheroids [thick black arrow] (1A) and residues specifically stained with fluorescent-labeled WGA (1B) and MAL-I (1C) [thin black arrow] [200X]

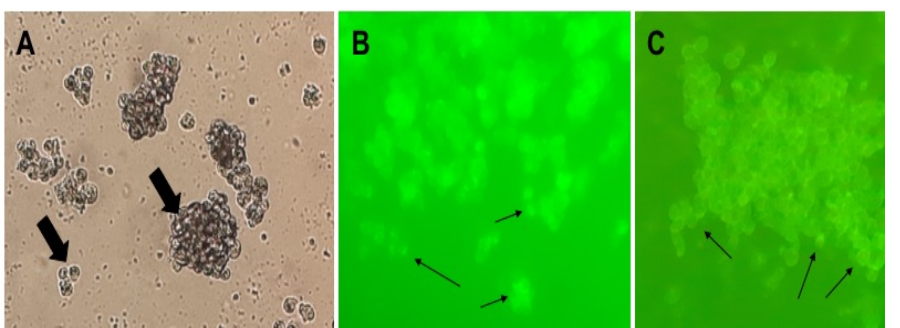

Fig. 2. Cells produced in MDA-MB-231 three-dimensional (3D) cell line, spheroids. Control cells and spheroids. Control cells and spheroids [thick black arrow] (1A) and residues specifically stained with fluorescent-labeled WGA (1B) and MAL-I (1C) [thin black arrow] [200X]
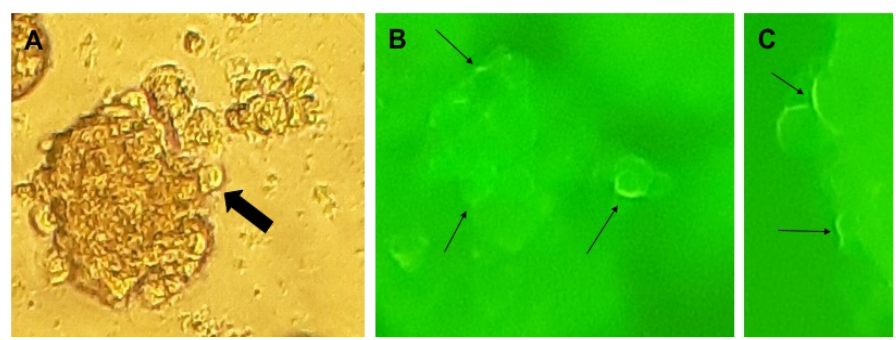

Fig. 3. Cells produced in MCF-7 three-dimensional (3D) cell line, spheroids. Control cells and spheroids [thick black arrow] (1A) and residues specifically stained with fluorescent-labeled WGA (1B) and MAL-I (1C) [thin black arrow] [400X]
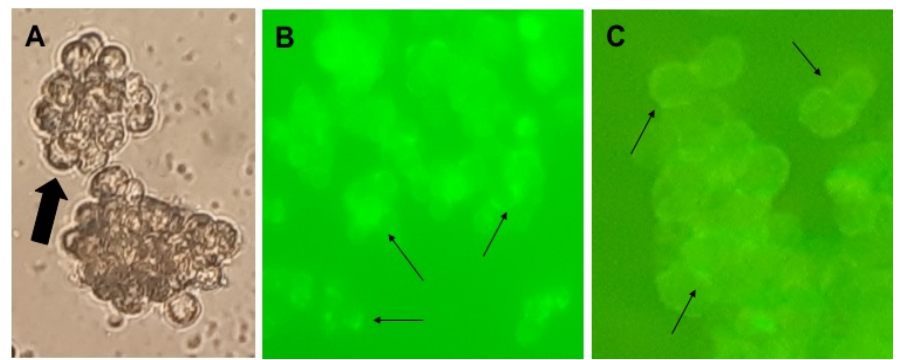

Fig. 4. Cells produced in MDA-MB-231 three-dimensional (3D) cell line, spheroids. Control cells and spheroids. Control cells and spheroids [thick black arrow] (1A) and residues specifically stained with fluorescent-labeled WGA (1B) and MAL-I (1C) [thin black arrow] [200X]

The internal evaluation between Fig. 1 and Fig. 3 revealed that MCF-7 [ER (+)] spheroids were intensely labeled with FITC-[WGA] lectin, however, this labeling was more intense at the surface level of the cells. We can state in other words that GlcNAc and Neu5Ac sialic acid units beside glycan units containing particularly $\beta-G l c N A c$ type sialic acid were found to be intense on the cell membranes of the $3 \mathrm{D}$ cancer cell lines.

The internal evaluation between Fig. 2 and Fig. 4 demonstrated that MDA-MB-231 [ER (-)] spheroids were intensely labeled by both FITC-[WGA] FITC-[MAL-I] lectins. The obvious difference on these images was that both GlcNAc and Neu5Ac sialic acid units beside glycan units containing particularly B-GlcNAc type sialic acid specifically labeled by FITC-[WGA] lectin and also glycan units containing particularly Galß4GlcNAc type glycan units labeled by FITC[MAL-I] were not only focused on the cell membranes of the 3D cancer cell lines, but also, they were intensely encountered in the cytoplasm and functioning in particularly cell adherence and division. This result indicates that MDA-MB-231 [ER (-)] breast cancer cell line is a much more aggressive or metastatic cancer type. In addition, these outcomes obtained using spheroid cell culture method as the closest model to the patient sample suggest that the present study carried out based on different staining and radiation intensities with a two-hour lasting short staining procedure can be implemented as an easy, sensitive, and fast method in differentiation of two cancer types.

As a consequence, sialic acid (GlcNAc, Neu5Ac) units, particularly $\beta-G l c N A c$ type sialic acid glycan units and Galß4GlcNAc type glycan units were sensitively and 
specifically labeled by FITC-[WGA] and FITC-[MAL-I], and the results were illustrated. Thereby, a modified immunofluorescent staining method that can perform a fast, sensitive, and specific labeling was tested and developed in one of the closest cell models to the patient samples.

\section{Discussion}

Oligosaccharides and polysaccharides defined as glycans are the cell surface molecules located at the surfaces of all the cells including embryonic and pluripotent stem cells and constitute an intense glycocalyx layer as usually added to proteins and lipids. It has been determined that investigations conducted in the field of glycobiology have detected that these glycoconjugates have various and complicated biological functions. The cells respond to the environment circumstances thanks to this layer while this layer also regulates the interactions with neighboring cells. Beside these, glycoconjugates play an effective role in determination of cellular positioning (Lanctot and Varki, 2007; Patil et al., 2012; $\mathrm{Yu}$ et al., 2014). The change in the amounts of sialic acid residues particularly containing $\alpha-2,3$ - and $\alpha-2,6$ bonds located on the cell surfaces leads to development of avascular multicellular tumoral formations in normal and cancer cells. It has been demonstrated that they have a capability to form in vivo xenograft tumors particularly in the cancer cell lines. In the light of these data; it may be concluded that the ability to specifically label these xenograft tumor formations to differentiate cancer cells from the normal cells is highly important (Akasov et al., 2016).

Several studies have been carried out on specifically labeling of glycoconjugates using fluorescent-labeled lectins (Kataoka and Tavassoli, 1985; Fujitani et al., 2013; Ishihara et al., 2013), appropriate selection of the cells (Lawrence et al., 2014, Kawabe et al., 2013), their use in the oriented treatment (Sethuraman and Stadheim 2006, Abdelkrim et al., 2009) and treatment of many metabolic disorders as adjuvant therapies (Gangaram-Panday et al., 2007; Varki, 2008) and these studies are currently continuing.

Whelan et al. (2009) have reported their study on N-linked membrane glycoproteomics obtained from three breast cancer lines using hydrazide chemistry-based enrichment. They used ER and PR (+) and Her2 (-) MCF-7, ER and PR (-) and Her2 (+) MDAMB-453 and ER (-); PR (-) and Her2 (-) (triple negative) MDA-MB-468 cell strains. In that study, the cells that constitute ER (+) MCF-7 and ER (-) MDAMB-231 breast cancer cell lines were applied fluorescent (FITC)-labeled Maackia amurensis lectin-1 (FITC-[MAL-I]) that can specifically bind to a kind of galactose (Galß4GlcNAc) and fluorescent (FITC)-labeled Wheat Germ Aglutinin (FITCTriticum vulgaris [WGA]) that can specifically bind to some sialic acid types (GlcNAc, Neu5Ac) and particularly B-type NAcetyl Glucosamine (B-GlcNAc) sialic acid glycan. By this process, the differences between the amounts of sugar residues detected on membrane surfaces of mentioned ER (+) MCF-7 and ER (-) MDAMB-231 breast cancer cell lines were specifically labeled and radiation intensities was relatively compared.

In the light of this information, the differences between the amounts of sugar residues detected on membrane surfaces of mentioned ER (+) MCF-7 and ER (-) MDAMB-231 breast cancer cell lines in our previous studies we have performed using 2D cell culture model (Mater and Özdaş 2018; Demircan and Mater, 2019) were specifically labeled with FITC-[WGA] and FITC-[MAL-I] and radiation intensities was relatively compared (Figs. 1-4). Accordingly, three-dimensional (3D) spheroid models were created from the MCF-7 and MDA-MB 231 breast cancer cell lines (Fig. 1A and Fig. 2A). MCF-7 [ER $(+)$ ] and MDA-MB-231 [ER (-)] spheroids were stained with fluorescent-labeled lectins (Fig. 1B-C and Fig. 2B-C). Correspondingly; sialic acid units (GlcNAc, Neu5Ac) and also glycan units containing particularly B-GlcNAc type sialic acids were visualized by using FITC-[WGA] while Galß4GlcNAc type glycan units were displayed by applying FITC-[MAL-I] as the specifically binding fluorescent-labeled lectins. Their binding ability and the fact that this method was applicable also in the $3 \mathrm{D}$ cell model as the closest design to the patient samples were manifested (Figs. 1 and 2).

The internal evaluation of the breast cancer cell lines with different characteristics (Figs. 1 and 3) revealed that spheroids created by MCF-7 [ER (+)] cells were intensely labeled by FITC-[WGA] lectin, however, this labeling was mostly concentrated on the membrane surfaces of the cells (Figure 1B and $3 \mathrm{~B})$. We can state in other words that GlcNAc, Neu5Ac sialic acid units and particularly glycan units containing $\mathrm{B}$ GlcNAc type sialic acid specifically labeled with FITC[WGA] lectin was intensely found in (3D) breast cancer cell lines. Contrarily, Galß4GlcNAc type glycan units specifically labeled applying FITC-[MAL-I] were detected on the cell membrane in the same manner, however, their amount was found to be very much lower than the residues specifically labeled with FITC-[WGA] lectin (Figs. 1C and 3C). On the other hand, the internal evaluation between the spheroids created by MDA-MB-231 [ER (-)] cells (Figs. 2 and 4) demonstrated that spheroids were intensely stained by both FITC-[WGA] (Figs. 2B and 4B) and FITC-[MAL-I] lectins (Figs. $2 \mathrm{C}$ and $4 \mathrm{C}$ ). The obvious difference on these images was that both GlcNAc and Neu5Ac sialic acid units beside glycan units containing particularly $B$-GlcNAc type sialic acid specifically labeled by FITC-[WGA] lectin and also glycan units containing particularly Galß4GlcNAc type glycan units labeled by FITC-[MAL-I] were not only focused on the cell membranes of the 3D cancer cell lines, but also they were intensely encountered in the cytoplasm and functioning in particularly cell adherence and division (Figs. 2B-C and 4BC). This result indicates by the micrographs that MDA-MB231 [ER (-)] breast cancer cell line included much more sialic acid units and is a much more aggressive or metastatic cancer type. 
In addition, these outcomes obtained using spheroid cell culture method as the closest model to the patient sample proved once more that assessment of the sugar units can be implemented as a relatively easier, absolutely sensitive and fast method in differentiation of two cancer types thanks to the present study carried out based on different staining and radiation intensities with a two-hour lasting short staining procedure.

It has been exhibited by the present study that it would be possible to label the sugar units found on the surfaces of breast cancer cells with specifically binding lectins and thus to identify the sugar profiles of the normal and different breast cancer cells. Additionally, it has been also clarified that it would possible to determine the structure of the monomer units that constitute these specifically detected residues and that the impact of the sugar molecules that have a vital function in recognition-adherence mechanism of the cells on the breast cancer cell types may be analyzed visually and by micrographs.

3D cell culture models applying successfully designed and improved lectin-labeling method will allow visualization with fluorescent microscopes as well as achievement and evaluation of the closest accurate results to the living tissue. Our present and further studies applying improved and improvable specific labeling methods will provide a precious contribution to the research and healthcare sector in "development of fastresulting alternative immunohistochemical labeling method/methods".

As a conclusion, the most crucial feature of the present study is that the differences between sugar units on the cell surface were identified in the three-dimensional spheroid cell culture model that shows the closest similarity to the living tissue using fluorescent-labeled lectin for the fast, sensitive, and specific labeling method (Mater and Özdaş, 2018; Demircan and Mater, 2019) of which preliminary studies were performed and modified by us. The surface sugar profiles of two different breast cancer cell lines were analyzed by this method. The inability to specifically label the residues have provided guiding information in both early diagnosis of breast cancer and also oriented drug studies. Thus, collection of essential information for a study has been started to develop a technique allowing a fast, sensitive and specific labeling to be implemented to the closest cell form regarding living tissue and to obtain kit and patent right.

\section{Conflict of interest}

None to declare.

\section{Acknowledgments}

None to declare.

\section{References}

1. Abdelkrim, H., Dominguez-Bendala, J., Wakeman, J., Arredouani, M., Soria, B., 2009. The immune boundaries for stem cell-based therapies: Problems and prospective solutions. J. Cell. Mol. Med. 13, 1464-1475.

2. Akasov, R., Haq, S., Haxho, F., Samuel, V., Burov, S. V.,
Markvicheva, E., Neufeld, R. J., Szewczuk, M.R., 2016. Sialylation transmogrifies human breast and pancreatic cancer cells into 3D multicellular tumor spheroids using cyclic RGDpeptide induced self-assembly. Oncotarget. 7, 66119-66134.

3. Anthony, S., Leong, Y., Zhuang, Z., 2011. The changing role of pathology in breast cancer diagnosis and treatment. Pathobiology. 78, 99-114.

4. Demircan, G., Mater, Y., 2019. Effects of Fluorescent Marked Maackia Amurensis-Lectin-1 and Wheat Germ Aglutin on the Cell Surface Glycan Profiles in Two Different Breast Cancer Cell Lines. İstanbul Tıp Fakültesi Dergisi, 82(2), 1-7.

5. Eccles, S. A., Aboagye, O. E., Ali, S., Anderson, S. A., Armes, J., Berditchevski, F., Blaydes, J. P., Brennan, K., Brown, J. N., Bryant, H. E., et al. 2013. Critical research gaps and translational priorities for the successful prevention and treatment of breast cancer. Breast Cancer Research. 15, 92.

6. Fujitani, N., Furukawa, J. I., Araki, K., Fujioka, T., Takegawa, Y., Piao, J., Nishioka, T., Tamura, T., Nikaido, T., Ito, M., Nakamura, Y., Shinohara, Y., 2013. Total cellular glycomics allows characterizing cells and streamlining the discovery process for cellular biomarkers. PNAS. 110-6, 2105-2110.

7. Gangaram-Panday, S., Faas, M. M., Vos, P., 2007. Towards stem cell therapy in the endocrine pancreas. TRENDS in Molecular Medicine. 13, 4.

8. Ishihara, T., Kakiya, K., Takahashi, K., Miwa, H., Rokushima, M., Yoshinaga, T., Tanaka, Y., Ito, T., Togame, H., Takemoto, H., Amano, M., Iwasaki, N., Minami, A., Nishimura, S. I., 2013. Discovery of novel differentiation markers in the early stage of chondrogenesis by glycoform-focused reverse proteomics and genomics. Biochimica. Biophysica. Acta. 27758: p1 1; 4C:3-9.

9. Kataoka, M., Tavassoli, M., 1985. Identification of lectin like substances recognizing galactosyl residues of glycoconjugates on the plasma membrane of marrow sinüs endothelium. Blood. $65,1163-1171$.

10. Kawabe, K., Tateyama, D., Toyoda, H., Kawasaki, N., Hashii, N., Nakao, H., Matsumoto, S., Nonaka, M., Matsumura, H., Hirose, Y., Morita, A., Katayama, N., Sakuma, M., Kawasaki, N., Furue, M. K., Kawasaki, T., 2013. A novel antibody for human induced pluripotent stem cells and embryonic stem cells recognizes a type of keretan sulfate lacking over sulfated structures. Glycobiology. 23(3), 322-336.

11. Kohnz, R. A., Roberts, L. S., Detomaso, D., Bideyan, L., Yan, P., Bandyopadhyay, S., Goga, A., Yosef, N., Nomura, D. K. 2016. Protein sialylation regulates a gene expression signature that promotes breast cancer cell pathogenicity. ACS Chemical Biology. 11(8), 2131-2139.

12. Lanctot, P. M., Gage, F.H., Varki, A. P., 2007. The glycans of stem cells. Curr. Opin. Chem. Bio. 11, 373-380.

13. Lawrence, R., Brown, J. R., Lorey, F., Dickson, P. I., Crawford, B.E., Esko, J.D., 2014. Glcan based biomarkers for mucopolysaccharidoses. Mol. Genet. Metab. 111, 73-83.

14. Martinez-Duncker, I., Salinas-Marin, R., Martinez-Duncker, C., 2011. Towards in vivo imaging of cancer sialylation. Int. J. Mol. Imaging. 2011, 1-10.

15. Mater, Y., Beyhan-Özdas, S., 2018. The analysis of surface saccharide profiles through fluorescein-labelled lectins in a rat pancreatic tissue with established metabolic syndrome model. Turk. J. Biochem. 44(1), 98-104.

16. Nath, S., Devi, G. R., 2016. Three-dimensional culture systems in cancer research: focus on tumor spheroid model. Pharmacol. Ther. 163, 94-108. 
17. Patil, S.A., Chandrasekaran, E. V., Matta, K.L., Parikh, A., Tzanakakis, E. S., Neelamegham, S., 2012. Scaling down the size and increasing the throughput of glycosyltransferase assay: activity changes on stem cell differentiation. Analytical. Biochemistry. 425, 135-144.

18. Pinho, S.S., Reis, C.A., 2015. Glycosylation in cancer: Mechanisms and clinical implications. Nat. Rev. Can. 15(9), 540-555.

19. Samraj, A. N., Läubli, H., Varki, N., Varki, A., 2014. Involvement of a non-human sialic acid in human cancer. Front. Onco. 1-13.

20. Sethuraman, N., Stadheim, T.A., 2006. Challenges in therapeutic glycoprotein production. Curr. Opin. Biotechnol. 17, 341-346.

21. Song, E., Mechref, Y., 2015. Defining glycoprotein cancer biomarkers by MS in conjunction with glycoprotein enrichment. Biomark. Med. 9(9), 835-844.

22. Whelan, S. A., Lu, M., He, J., 2009. Mass spectrometry (LCMS/MS) site-mapping of N-glycosylated membrane proteins for breast cancer biomarkers. J. Proteome. Res. 8(8), 4151-4160.

23. Varki, A., 2017. Glycosylation Changes in Cancer. In Cold Spring Harbor (NY) $3^{\text {rd }}$ edition. 597-609.

24. Varki, A., 2008. Sialic acid in human health and disease. Trend Mol. Med. 14(8), 351-360.

25. Yu, C.C., Hill, T., Kwan, D.H., Cheng, H.M., Lin, C.C., Wakarchuk, W., Withers, S. G., 2014. A plate based high throughput activity assay for polysialyltransferase from Neisseria meningitidis. Anal. Biochem. 444, 67-74. 